\title{
Propionibacterium avidum
}

National Cancer Institute

\section{Source}

National Cancer Institute. Propionibacterium avidum. NCI Thesaurus. Code C86681.

A species of anaerobic, Gram-positive, rod shaped bacteria assigned to the phylum

Actinobacteria. This species is non-spore forming, catalase positive, indole negative,

hydrolyzes esculin, gelatin, and casein, but not starch, and does not reduce nitrate. P.

avidum was isolated from intertriginous skin areas, especially the axilla, and is associated with chronically infected sinuses, ulcers, and abscesses. 